\title{
Description of sub-barrier heavy ion fusion in a semiclassical quantum tunneling model
}

\author{
Nadia Yahlali* and José Díaz \\ Instituto de Física Corpuscular, Centro Mixto Universidad de Valencia-CSIC, Dr. Moliner, 50, E-46100 Burjasot, Spain \\ Taklit Sami \\ Institut de Physique, USTHB, Algiers, Algeria \\ (Received 18 July 1995)
}

\begin{abstract}
In this paper we apply the semiclassical method based on the Feynman path integral formalism to sub-barrier fusion of heavy nuclei. Cross sections are calculated and compared to experimental data and to coupledchannel calculations for different mass systems: ${ }^{32} \mathrm{~S}+{ }^{24} \mathrm{Mg},{ }^{58} \mathrm{Ni}+{ }^{64} \mathrm{Ni}$, and ${ }^{16} \mathrm{O}+{ }^{208} \mathrm{~Pb}$. The semiclassical method and coupled-channel calculations give comparable results. It is found that the coupling produces a renormalization of the barrier that is responsible for the enhancement of sub-barrier fusion cross sections and a dissipative force along the classical tunneling path.

PACS number(s): 25.70.Jj, 24.10.Eq, 21.60.Ev
\end{abstract}

\section{INTRODUCTION}

Sub-barrier fusion of heavy ions has motivated many theoretical and experimental investigations in the years since the observation of a large enhancement of experimental fusion cross sections with respect to the predictions of the onedimensional barrier penetration model [1]. Significant isotopic effects for different projectile and target combinations in sub-barrier fusion cross sections have also been observed [2]. Different theoretical models, which include additional degrees of freedom of the fusing system, have been elaborated to explain these observations. Among the main ones are (i) deformed or vibrating potential models $[3,4]$; (ii) optical potential models in which a part of the imaginary potential is associated to fusion [5]; (iii) coupled-channel models in which the excited states of each nucleus are treated in a full quantum mechanical way leading to the solution of a set of coupled Schrödinger equations [6]; (iv) approximations based on the Feynman path-integral method, in which the effect of additional degrees of freedom on the relative motion is formulated in the influence functional formalism $[7,8]$; (v) models which invoke collective phenomena as neutron flow or neck formation $[9,10]$. Coupled-channel models are in principle well founded and accurate for treating multidimensional tunneling, the input required is only the level scheme and some properties of the relevant excited states of the fusing nuclei together with optical potentials and form factors fitted to experimental cross sections. However, in practice only a few inelastic states can be included in the calculations due to the fast increase of computing time with the number of coupled states and to numerical instabilities arising in large sets of coupled equations $[11,12]$. Moreover when one wants to include degrees of freedom of a very different nature as transfer or deep inelastic reactions, the problem becomes quite difficult to solve by numerical coupled-channel methods because of the nonorthogonality and different range of excitation energies of the channel states. This severely limits in practice the number of chan-

\footnotetext{
*On leave from Institut de Physique, USTHB, Algiers, Algeria.
}

nels that can be treated simultaneously. In the path integral method, however, each different degree of freedom gives an independent contribution to the influence functional. In addition, the computational efforts grow slowly with the dimensionality of the system. This makes the path integral method convenient to treat on the same footing single nucleus excitations and complex mechanisms as transfer or deep inelastic. Thus the path integral method, although insufficiently developed, offers good possibilities for studying fusion coupled to degrees of freedom of a very different nature. Some analytical results have been obtained in the literature $[8,13]$, but to our knowledge either realistic numerical calculations of cross sections or comparisons to other methods to evaluate the real power of this method do not exist. The purpose of this paper is to present detailed numerical calculations of sub-barrier fusion cross sections based on the influence functional formalism for some different systems $\left({ }^{32} \mathrm{~S}+{ }^{24} \mathrm{Mg},{ }^{58} \mathrm{Ni}+{ }^{64} \mathrm{Ni}\right.$, and $\left.{ }^{16} \mathrm{O}+{ }^{208} \mathrm{~Pb}\right)$ for which there exist measurements of both elastic and fusion cross sections. Our results are compared to experimental data and to calculations performed with the widely employed simplified coupled-channel code CCFUS [14]. We also discuss such features of the coupled-tunneling process as adiabaticity, the effect of coupling before entrance into the barrier, and dissipative reflections of the trajectory under the barrier.

\section{DESCRIPTION OF THE MODEL}

We consider in this paper only inelastic excitations, which we assume to be well described by harmonic oscillators that form the "intrinsic" system. This system is linearly coupled to the nucleus-nucleus relative motion coordinate $R$, called "collective." We consider in the calculations of the influence functional the effects of the coupling before tunneling, which we account for by the intrinsic state of the fusing system at the entrance of the barrier.

We give below the expressions for only one oscillator; those for several oscillators are obtained by a simple addition. The Hamiltonian for the intrinsic coupled system is

$$
H_{\mathrm{int}}=\hbar \omega a^{+} a+\sigma f(R)\left(a^{+}+a\right)
$$


where $\sigma$ is the deformation length of the excited state [28] and $f(R)$ the coupling form factor. The time-evolution operator of the intrinsic system can be cast in the form [17]

$$
\hat{U}(t, 0)=e^{-i a^{+} a \omega t} e^{A(t)} e^{-B^{*}(t) a^{+}} e^{B(t) a},
$$

where

$$
\begin{gathered}
B(t)=-\frac{i \sigma}{\hbar} \int_{0}^{t} f\left[R\left(t^{\prime}\right)\right] e^{-i \omega t^{\prime}} d t^{\prime}, \\
A(t)=-\left(\frac{\sigma}{\hbar}\right)^{2} \int_{0}^{t} f\left[R\left(t^{\prime}\right)\right] e^{-i \omega t^{\prime}} d t^{\prime} \int_{0}^{t^{\prime}} f\left[R\left(t^{\prime \prime}\right)\right] e^{i \omega t^{\prime \prime}} d t^{\prime \prime} .
\end{gathered}
$$

The intrinsic state of the system at very large radial distances is the vacuum state $|0\rangle$ which is the direct product of the ground states of both nuclei. When the nuclei approach within the range of the coupling form factor, the intrinsic system couples to the radial motion. The intrinsic state at a given time $t$ is then given by

$$
|\alpha(t)\rangle=\hat{U}(t, 0)|0\rangle=e^{A(t)} e^{\alpha(t) a^{+}}|0\rangle,
$$

with

$$
\alpha(t)=-B^{*}(t) e^{-i \omega t}=-\frac{i \sigma}{\hbar} e^{-i \omega t} \int_{0}^{t} f\left[R\left(t^{\prime}\right)\right] e^{i \omega t^{\prime}} d t^{\prime} .
$$

It is seen that an intrinsic state coupled linearly evolves as a coherent state $[15,16]$ when the nuclei approach the barrier. It is this coherent state which undergoes tunneling when the nuclei reach the external turning point of the barrier.

The vanishing of the coupling when the nuclei are far away allows us to rewrite $\alpha(t)$ at the external barrier $R_{E}$ as an adiabatic value and a shift from adiabaticity,

$$
\alpha_{i}=\alpha_{i a d}+\gamma,
$$

where the adiabatic parameter $\alpha_{i a d}$ and the shift from adiabaticity $\gamma$ are given by Müller and Takigawa [17]:

$$
\begin{gathered}
\alpha_{i a d}=-\frac{\sigma f\left(R_{E}\right)}{\hbar \omega}, \\
\gamma=\frac{\sigma}{\hbar \omega} e^{-i \omega t\left(R_{E}\right)} \int_{0}^{t\left(R_{E}\right)}\left(\frac{d f}{d R}\right)_{t^{\prime}} \dot{R}\left(t^{\prime}\right) e^{i \omega t^{\prime}} d t^{\prime} .
\end{gathered}
$$

In the adiabatic process, the intrinsic system is coupled to the relative motion but remains in the ground state. We neglect the shift $\gamma$, and assume that at the entrance of the barrier the ground state is the adiabatic coherent state

$$
\left|\alpha_{i a d}\right\rangle=e^{-\left|\alpha_{i a d}\right|^{2} / 2} e^{\alpha_{i a d} a^{+}}|0\rangle,
$$

which reduces to the usual ground state for $\alpha_{i}=0$. The inclusive influence functional is determined by using the completeness relation of the coherent states $[15,16]$ and the imaginary time procedure for tunneling [7]. The penetrability is calculated along the classical trajectory obtained by solving the classical equation of motion

$$
\mu \ddot{R}=\frac{d V}{d R}+F(\tau)
$$

where the force $F(\tau)$ is given by

$$
\begin{aligned}
F(\tau)= & -\frac{\sigma^{2}}{\hbar}\left(\frac{d f}{d R}\right){ }_{\tau}\left[e^{-\omega \tau} \int_{0}^{\tau} f\left[R\left(\tau_{1}\right)\right] e^{\omega \tau_{1}} d \tau_{1}\right. \\
& +e^{\omega \tau} \int_{\tau}^{\theta} f\left[R\left(\tau_{1}\right)\right] e^{-\omega \tau_{1}} d \tau_{1} \\
& \left.+e^{\omega(\tau-2 \theta)} \int_{0}^{\theta} f\left[R\left(\tau_{1}\right)\right] e^{\omega \tau_{1}} d \tau_{1}\right]+\sigma \alpha_{i}\left(\frac{d f}{d R}\right)_{\tau} \\
& \times\left(e^{-\omega \tau}+e^{\omega(\tau-2 \theta)}\right),
\end{aligned}
$$

where $\mu$ is the reduced mass of the system and $\theta$ the tunneling time along the classical trajectory. Integrating by parts Eq. (11), Eq. (10) can be rewritten in the form

$$
\mu \ddot{R}=\frac{d V_{\mathrm{eff}}(R)}{d R}+F_{D}(\tau),
$$

where the effective barrier $V_{\text {eff }}$ is usually called the adiabatic barrier, given by

$$
V_{\mathrm{eff}}(R)=V(R)-\frac{[\sigma f(R)]^{2}}{\hbar \omega} .
$$

The force $F_{D}(\tau)$ is given by

$$
\begin{aligned}
F_{D}(\tau)= & \frac{\sigma^{2}}{\hbar \omega}\left(\frac{d f}{d R}\right){ }_{\tau} e^{-\omega \tau} \int_{0}^{\tau}\left(\frac{d f}{d R}\right)_{\tau_{1}} \dot{R}\left(\tau_{1}\right) e^{\omega \tau_{1}} d \tau_{1} \\
& -e^{\omega \tau} \int_{\tau}^{\theta}\left(\frac{d f}{d R}\right){ }_{\tau_{1}} \dot{R}\left(\tau_{1}\right) e^{-\omega \tau_{1}} d \tau_{1} \\
& \left.+e^{\omega(\tau-2 \theta)} \int_{0}^{\theta}\left(\frac{d f}{d R}\right)_{\tau_{1}} \dot{R}\left(\tau_{1}\right) e^{\omega \tau_{1}} d \tau_{1}\right] .
\end{aligned}
$$

It appears from Eq. (12) that the coupling of the nuclear degrees of freedom to the relative motion has two effects: (i) a renormalization of the barrier proportional to the inverse of the excitation energy of the state; (ii) the action along the dominant path of a non-Markovian force $F_{D}(\tau)$ that is in general of dissipative character. The penetrability of the barrier for a given energy $E$ in the center of mass is given by [18]

$$
P(E)=P_{R}(E) \cdot P_{\eta}(E),
$$

where $P_{R}(E)$ is the contribution to the penetrability along the classical path obtained by solving Eqs. (10) and (12) and $P_{\eta}(E)$ is the contribution of fluctuations of the trajectory around the classical path, which is not considered in this paper. The penetrability $P(E)$ is then approximated by 


$$
\begin{aligned}
P(E) \approx & P_{R}(E)=\exp \left(-2 \int_{0}^{\theta}\left[\frac{\mu \dot{R}^{2}}{2}+U(R)\right.\right. \\
& \left.\left.+W_{R}(\tau)-E\right] \frac{d \tau}{\hbar}\right)
\end{aligned}
$$

where $W_{R}$ is the influence potential given by

$$
\begin{aligned}
W_{R}(\tau)= & -\frac{\sigma^{2}}{\hbar}\left(1+e^{-2 \omega(\theta-\tau)}\right) f[R(\tau)] e^{-\omega \tau} \\
& \times \int_{0}^{\tau} f\left[R\left(\tau_{1}\right)\right] e^{\omega \tau_{1}} d \tau_{1}+2 \alpha_{i}\left(\hbar \omega e^{-2 \omega \tau} \alpha_{i}\right. \\
& \left.+\sigma f[R(\tau)]\left(e^{-\omega \tau}+e^{\omega(\tau-2 \theta)}\right)\right) .
\end{aligned}
$$

The fusion cross section is calculated from the partial wave expansion

$$
\sigma_{f}(E)=\frac{\pi}{k^{2}} \sum(2 \ell+1) P \ell(E)
$$

where $k$ is the asymptotic momentum. In the present calculations we evaluate quantitatively the effect on the subbarrier fusion cross section of the renormalization of the barrier and the force $F_{D}$ separately. We calculate first the fusion cross sections using the Hill-Wheeler formula for the penetrabilities with the effective barrier given by Eq. (13),

$$
P(E)=\frac{1}{1+\exp \left(\frac{-2 \pi\left(E-V_{B}^{\mathrm{eff}}\right)}{\hbar \Omega_{\mathrm{eff}}}\right)},
$$

where $V_{B}^{\text {eff }}$ is the height of the effective barrier and $\hbar \Omega_{\text {eff }}$ its curvature. To calculate the classical path we have numerically solved Eq. (10) by the Runge-Kutta method of fourth order. As the equation is an integro-differential one, with the force depending on the solution for the trajectory, we have used a self-consistent procedure, taking the trajectory with a vanishing force as the initial solution. We have found that the iterative process converges rapidly and usually five or fewer iterations are needed. The external turning point has been taken as the solution of $V(R)=E$, but due to the dissipation of the energy along the tunneling trajectory, the internal turning point differs from that of the classical trajectory without dissipation and has been taken as the point at which the velocity vanishes. This procedure has been done for each partial wave, stopping the calculations when the penetrability becomes negligible. In our calculations of the penetrabilities, we have considered the multiple reflections of the trajectory under the barrier by using the uniform approximation for the penetrability [19] derived for the nondissipative process

$$
P_{\text {unif }}(E)=\frac{P(E)}{1+P(E)} .
$$

For the dissipative case with which we are concerned, the action at each reflection can be different due to dissipation, and numerical integration along every one of the possible multiple-reflection trajectories should be performed to take this into account. We assume, however, that the actions along
TABLE I. Parameters of the optical potential for the different systems.

\begin{tabular}{lccc}
\hline \hline System & $V_{0}(\mathrm{MeV})$ & $r_{V}(\mathrm{fm})$ & $a_{V}(\mathrm{fm})$ \\
\hline${ }^{32} \mathrm{~S}+{ }^{24} \mathrm{Mg}$ & 340.47 & 1.010 & 0.598 \\
${ }^{16} \mathrm{O}+{ }^{208} \mathrm{~Pb}$ & 37.66 & 1.290 & 0.361 \\
${ }^{58} \mathrm{Ni}+{ }^{64} \mathrm{Ni}$ & 82.30 & 1.149 & 0.63 \\
\hline \hline
\end{tabular}

the reflected dissipative trajectories have very close values, and we use the above formula to obtain a first-order quantitative evaluation.

\section{APPLICATION OF THE MODEL TO EXPERIMENTAL DATA}

In order to compare the results of this model to other models and to experimental data we have chosen some nuclear systems for which there are available data of elastic and fusion cross sections simultaneously. The nuclear part of the interaction potential $V(R)$ in Eq. (10) has been taken as the real part of the optical potential parametrized in the Woods-Saxon form

$$
V_{N}(R)=-\frac{V_{0}}{1+\exp \left(\frac{R-R_{V}}{a_{V}}\right)}
$$

with parameters fitted to the elastic cross sections with the code ECIS [20]. The systems chosen have been ${ }^{32} \mathrm{~S}+{ }^{24} \mathrm{Mg}$ $[21,22],{ }^{16} \mathrm{O}+{ }^{208} \mathrm{~Pb}[23,24]$, and ${ }^{58} \mathrm{Ni}+{ }^{64} \mathrm{Ni}[25,27]$. The parameters of the Woods-Saxon potential for the three systems are given in Table I. The coupling form factor $f(R)$ has been taken as the derivative of the Woods-Saxon potential plus the Coulomb form factor

$$
\begin{aligned}
f(R)= & -\frac{d V_{N}(R)}{d R}+\frac{3 Z_{T} Z_{P} e^{2}}{2 \lambda+1}\left[\frac{R_{C}^{\lambda-1}}{R^{\lambda+1}} s^{\lambda-1} \theta\left(R-R_{C}\right)\right. \\
& \left.+\frac{R^{\lambda}}{R_{C}^{\lambda+2}} s^{\lambda-1} \theta\left(R_{C}-R\right)\right],
\end{aligned}
$$

where $Z_{P}, Z_{T}$ are the charges of the projectile and target, $\lambda$ the spin of the excited state, and $R_{C}$ the equilibrium radius of the charge density. The coefficient $s$ takes into account the $\beta R$ scaling $[26,28]$ which requires the nuclear and electromagnetic deformation lengths to be equal. The coupling parameter $\sigma$ is given in terms of the nuclear deformation length $\beta_{N} R_{V}$ by

$$
\sigma=\frac{\beta_{N} R_{V}}{\sqrt{4 \pi}}
$$

and the nuclear deformation parameters have been obtained from the electromagnetic ones $\beta_{\lambda}[21,29,30]$ by $\beta R$ scaling. The excited states considered for each nucleus and their respective deformation parameters are given in Table II.

At energies above the barrier $V_{B}^{0}$, the penetrabilities have been calculated without taking the coupling into account, by 
TABLE II. Deformation parameters for the excited states considered.

\begin{tabular}{llcc}
\hline \hline Nucleus & $J^{\pi}$ & $E_{\text {ex }}(\mathrm{MeV})$ & $\beta_{\lambda}$ \\
\hline${ }^{58} \mathrm{Ni}$ & $2^{+}$ & 1.454 & 0.216 \\
& $3^{-}$ & 4.475 & 0.186 \\
\cline { 2 - 4 } & $2^{+}$ & 1.344 & 0.198 \\
${ }^{64} \mathrm{Ni}$ & $3^{-}$ & 3.560 & 0.180 \\
\cline { 2 - 4 } & $2^{+}$ & 6.920 & 0.352 \\
${ }^{16} \mathrm{O}$ & $3^{-}$ & 6.130 & 0.712 \\
\cline { 2 - 4 } & $3^{-}$ & 2.614 & 0.120 \\
${ }^{208} \mathrm{~Pb}$ & $2^{+}$ & 4.086 & 0.055 \\
\cline { 2 - 4 } & $2^{+}$ & 2.230 & 0.312 \\
${ }^{32} \mathrm{~S}$ & $2^{+}$ & 1.369 & 0.505 \\
${ }^{24} \mathrm{Mg}$ & & & \\
\hline \hline
\end{tabular}

means of the Hill-Wheeler formula with the height $V_{B}^{0}$ and curvature $\hbar \Omega_{0}$ of the bare barrier.

We have found that at sub-barrier energies the imaginarytime method for tunneling breaks down when we move to energies very close to the barrier, typically between the adiabatic and bare barriers. The barrier width has very small values at these energies, usually less than one fermi, and the penetrabilities calculated from classical trajectories have unphysical values. This is due to the fact that the influence potential makes the barrier lower than the incident energy. To tackle this problem we have taken the penetrability as 0.5 at the bare barrier and interpolated to the region in which the semiclassical tunneling calculations work. The energy region in which this problem arises is narrow as can be seen from Table III in which the bare and renormalized adiabatic barriers are given for partial waves $\ell=0,8$, and 16 for each of the systems studied.

The way in which we have carried out this interpolation is to fit the numerical penetrabilities obtained in our semiclassical quantum tunneling model (SQT) to the Hill-Wheeler formula, obtaining $X(E)=\left(V_{B}-E\right) / \hbar \Omega$ values which reproduce these penetrabilities. These empirical $X(E)$ values have been fitted by a fourth-order polynomial vanishing at

TABLE III. Values of the bare and adiabatic barriers for $\ell=0$, 8 , and 16 for the three systems studied.

\begin{tabular}{lcccc}
\hline \hline System & $\ell$ & $V_{B}^{0}(\mathrm{MeV})$ & $V_{B}^{\text {eff }}(\mathrm{MeV})$ & $\hbar \Omega_{0}(\mathrm{MeV})$ \\
\hline${ }^{32} \mathrm{~S}+{ }^{24} \mathrm{Mg}$ & 0 & 28.090 & 27.691 & 3.78 \\
& 8 & 29.395 & 28.909 & 3.95 \\
& 16 & 33.098 & 32.333 & 4.40 \\
\cline { 2 - 5 }${ }^{58} \mathrm{Ni}+{ }^{64} \mathrm{Ni}$ & 0 & 100.109 & 97.780 & 3.97 \\
& 8 & 100.554 & 98.183 & 3.96 \\
& 16 & 101.795 & 99.304 & 4.00 \\
\cline { 2 - 5 }${ }^{16} \mathrm{O}+{ }^{208} \mathrm{~Pb}$ & 0 & 77.231 & 75.897 & 6.50 \\
& 8 & 77.940 & 76.578 & 6.49 \\
& 16 & 79.963 & 78.470 & 6.62 \\
\hline \hline
\end{tabular}

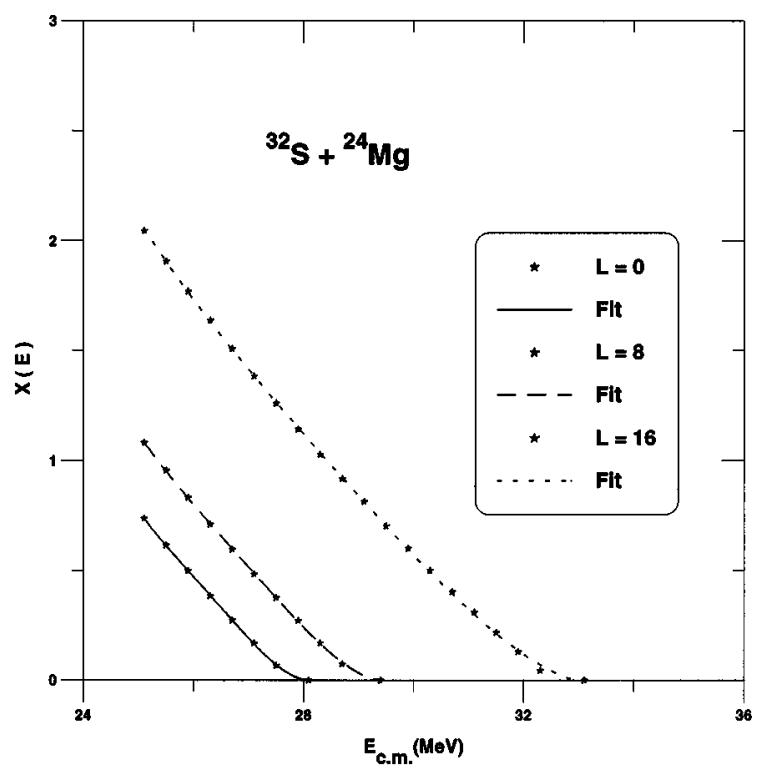

FIG. 1. System ${ }^{32} \mathrm{~S}+{ }^{24} \mathrm{Mg}$. The numerical values of $X(E)=\left(V_{B}-E\right) / \hbar \Omega$ are represented (stars) for the partial waves $\ell=0, \ell=8$, and $\ell=16$. Polynomial fits of order 4 are performed to deduce the penetrabilities between the renormalized and the bare barriers of each partial wave (the two stars at higher energy).

$E=V_{B}^{0}$. It may be considered that the energy dependence of this effective $X(E)$ takes into account the coupling effects. It is plotted for the systems and partial waves of Table III in Fig. 1, Fig. 2, and Fig. 3. A close analysis of $X(E)$ values reveals that below the adiabatic barrier they have a quadratic dependence on energy. In order to match smoothly the penetrabilities between the adiabatic and the bare barrier we needed to assume that $X(E)$ depends as a fourth-order polynomial on energy, reflecting a more involved nature of the coupling in this energy region. It is seen in Fig. 1, Fig. 2, and Fig. 3 that at energies far below the $s$-wave barrier, $X(E)$ depends quasilinearly on energy, indicating that the use of a

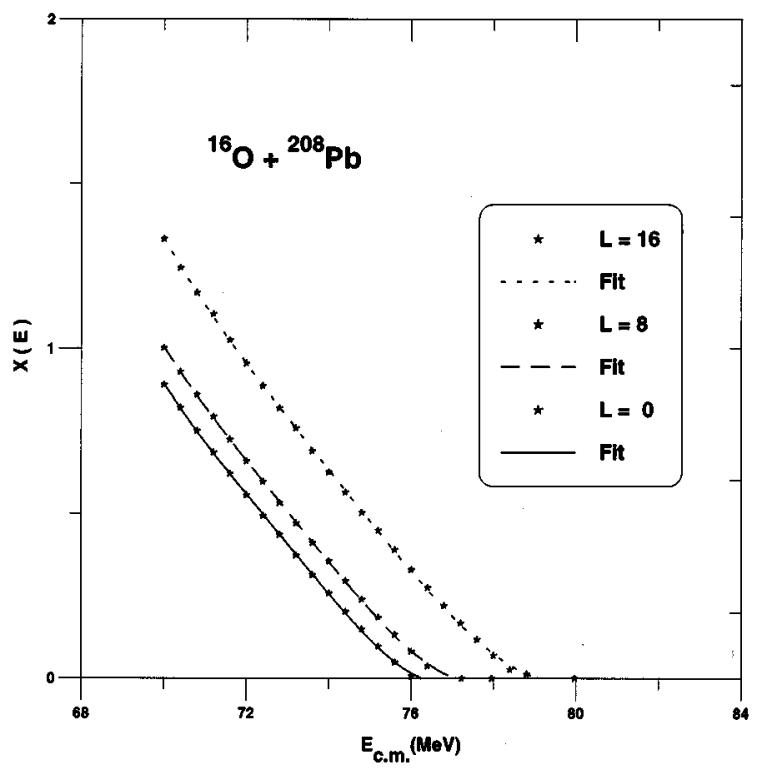

FIG. 2. Same as Fig. 1 for the system ${ }^{16} \mathrm{O}+{ }^{208} \mathrm{~Pb}$. 


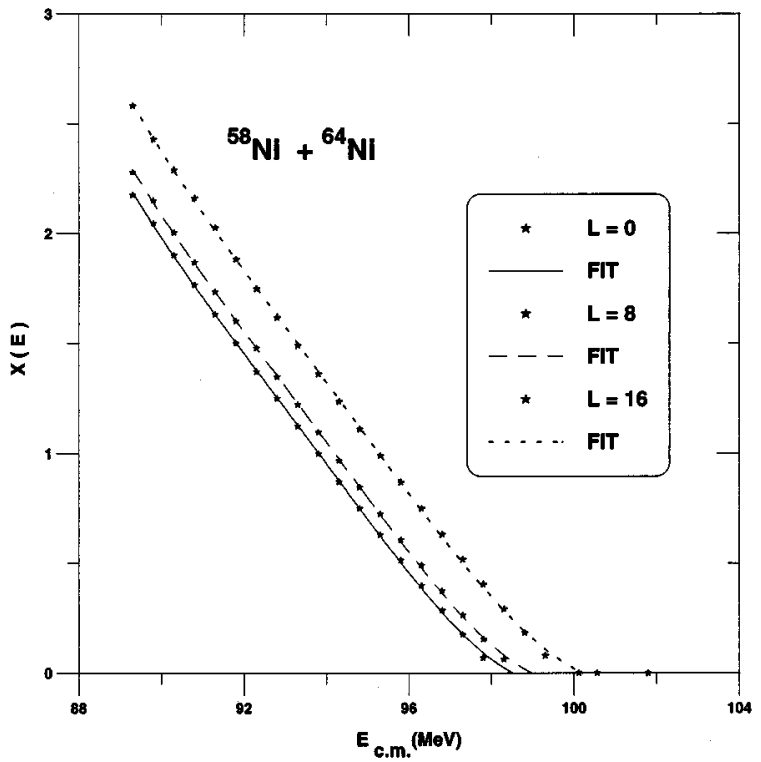

FIG. 3. Same as Fig. 1 for the system ${ }^{58} \mathrm{Ni}+{ }^{64} \mathrm{Ni}$.

renormalized barrier independent of energy would be a useful approximation in this region. This is no longer valid when we approach the barrier.

We have calculated the fusion cross sections for all energies from below to above the fusion barrier, using the SQT penetrabilities below the adiabatic barrier, the interpolated penetrabilities between the adiabatic and the bare barrier, and the Hill-Wheeler formula above the bare barrier. In Fig. 4, Fig. 5, and Fig. 6 the calculations performed with the total force $F(\tau)$ given in Eq. (11) are compared to calculations performed with the adiabatic potential of Eq. (13), and with

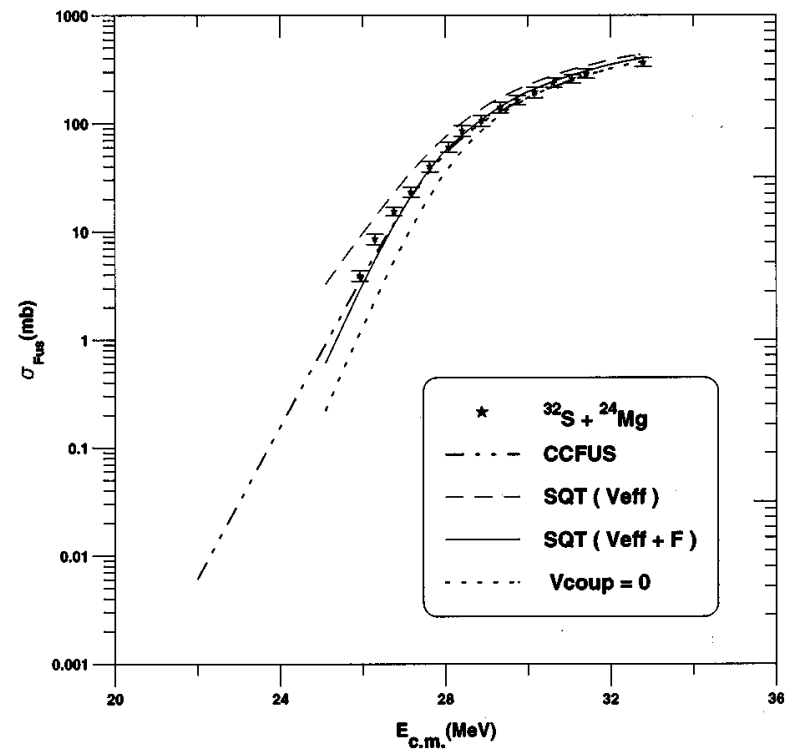

FIG. 4. Fusion cross sections for the system ${ }^{32} \mathrm{~S}+{ }^{24} \mathrm{Mg}$. The cross sections in the semiclassical quantum tunneling model with dissipation (full line) and without dissipation (long dashed line) are compared to those calculated with the code CCFUs (dashed dotted line), to the unidimensional barrier penetration model (dotted line) and to experimental data (stars).

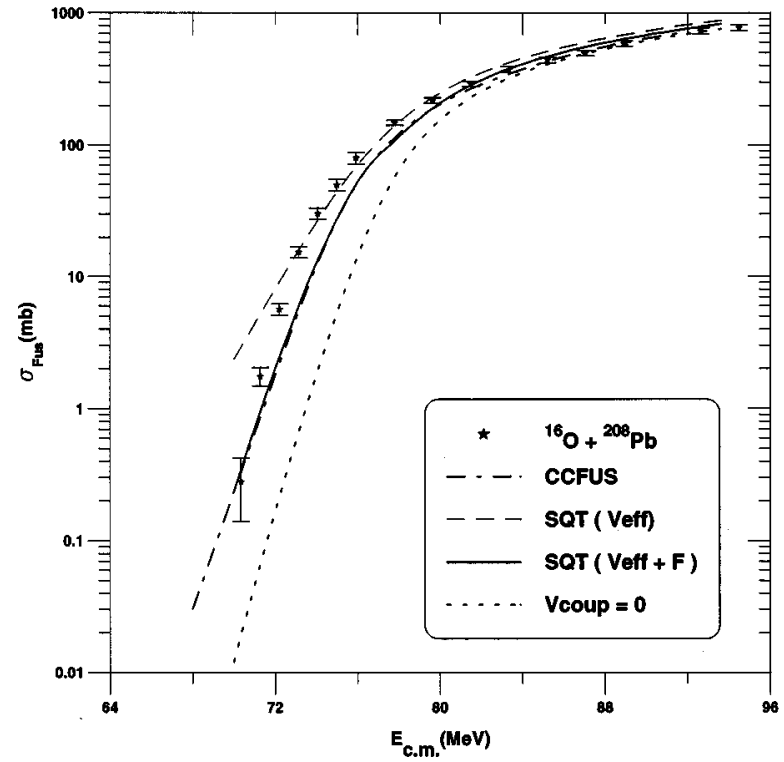

FIG. 5. Fusion cross sections for the system ${ }^{16} \mathrm{O}+{ }^{208} \mathrm{~Pb}$. The theoretical curves are represented as in Fig. 4.

the coupled channel code CCFUS [14]. The CCFUS and SQT calculations use the same input, with the same potentials and form factors. The initial state used in SQT calculations shown in the figures is, as in CCFUS, the initial ground state corresponding to a vanishing parameter $\alpha_{i}$. It can be observed in the figures that the renormalization has an important effect on the enhancement of the fusion cross sections, especially below the $s$-wave barrier energy. When the total force is considered the enhancement is less important, so we conclude that the action of the force $F_{D}(\tau)$ in Eq. (12) is to decrease the sub-barrier fusion cross sections with respect to the renormalization alone, and therefore is of dissipative character.

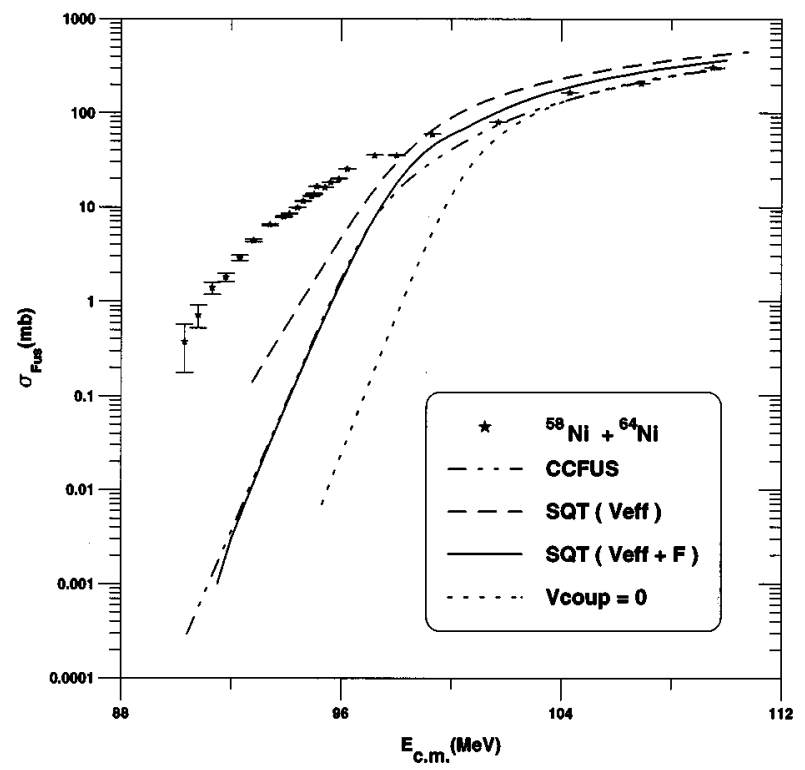

FIG. 6. Fusion cross sections for the system ${ }^{58} \mathrm{Ni}+{ }^{64} \mathrm{Ni}$. The theoretical curves are represented as in Fig. 4. 
It can be noted that the calculations done with the semiclassical approximation for linearly coupled tunneling agree quite well with the quantum mechanical calculations of CCFUS. At very low energies there are some discrepancies due mainly to the use of the parabolic approximation in CCFUS. In further calculations we have set the parameter $\alpha_{i}$ equal to its adiabatic value given by Eq. (7), finding that the fusion cross sections are not modified significatively with respect to those calculated with the unpolarized initial state. The coupling effects before and after the tunneling process do not seem to have a significant influence on the fusion cross sections in this model. We have also found that the use of the uniform approximation for the penetrabilities to take into account the multiple reflections of the trajectory under the barrier is a fair approximation at energies below the $s$-wave barrier for the systems studied. Nonuniform calculations of the penetrabilities do not seem to be, at this stage, of crucial need.

\section{CONCLUSIONS}

We have shown that the approach of path integrals for heavy-ion sub-barrier fusion gives results comparable to coupled-channel calculations for different systems. For in- elastic excitations of the nucleus, the renormalization effect is responsible for the enhancement of sub-barrier fusion cross sections. The important contribution of the dissipative force implies that the tunneling process for the systems considered is not adiabatic, although during the approach to the barrier entrance the adiabatic approximation is adequate. The coupling before tunneling which is accounted for by an initial coherent state appears to have a negligible influence on the fusion cross sections. We have also found, when multiple reflections are considered, that the uniform approximation can be used for the tunneling penetrabilities in the dissipative case with reasonable accuracy.

Our results allow us to conclude that the semiclassical calculations of the path integral for dissipative tunneling are sufficiently accurate for treating sub-barrier fusion at energies below the effective barrier. The importance of additional degrees of freedom as transfer or more complex ones as deep inelastic is evident in the case of $\mathrm{Ni}-\mathrm{Ni}$. These additional degrees of freedom in the path integral formalism will be treated in later works.

This paper has been supported by DGICYT under project PB90-0171.
[1] M. Beckerman, Phys. Rep. 129, 145 (1985). Rep. Prog. Phys. 51, 1047 (1988).

[2] R. A. Broglia, C. H. Dasso, S. Landowne, and G. Pollarollo, Phys. Lett. 133B, 34 (1983).

[3] H. Esbensen, Nucl. Phys. A352, 147 (1981).

[4] W. Reisdorf et al., Phys. Rev. Lett. 49, 1811 (1982).

[5] T. Udagawa, B. T. Kim, and T. Tamura, Phys. Rev. C 32, 124 (1985).

[6] M. J. Rhoades-Braun and P. Braun-Munzinger, Phys. Lett. 136B, 19 (1984).

[7] A. B. Balantekin and N. Takigawa, Ann. Phys. (N.Y.) 160, 441 (1985).

[8] P. Fröbrich, R. Lipperheide, and K. Möhring, Z. Phys. B 78, 325 (1990).

[9] P. H. Stelson, Phys. Lett. B 205, 190 (1988).

[10] A. Iwamoto and K. Harada, Z. Phys. A 326, 201 (1987).

[11] T. Tamura, Rev. Mod. Phys. 37, 679 (1965).

[12] C. H. Dasso, S. Landowne, and A. Winther, Nucl. Phys. A432, 495 (1985).

[13] G. F. Bertsh and N. Takigawa, Phys. Rev. C 29, 2358 (1984).

[14] C. H. Dasso and S. Landowne, Comput. Phys. Commun. 46, 187 (1987).

[15] R. J. Glauber, Phys. Rev. 131, 2766 (1963).

[16] W. H. Louisell, Quantum Statistical Properties of Radiation (Wiley, New York, 1990).

[17] B. Müller and N. Takigawa, Ann. Phys. (N.Y.) 173, 163 (1987).

[18] S. Y. Lee and N. Takigawa, Phys. Rev. C 28, 1323 (1983).
[19] D. M. Brink and U. Smilansky, Nucl. Phys. A405, 301 (1983).

[20] J. Raynal, Phys. Rev. C 23, 2571 (1981).

[21] G. M. Berkowitz, P. Braun-Munzinger, J. S. Karp, R. H. Freifelder, T. R. Renner, and H. W. Wilschut, Phys. Rev. C 28, 667 (1983).

[22] J. Díaz, J. L. Ferrero, F. Sánchez, B. Bilwes, and R. Bilwes, in Heavy Ion Interaction around the Coulomb Barrier, edited by C. Signorini, S. Skorka, P. Spolaore, and A. Vitturi (SpringerVerlag, Berlin, 1988).

[23] J. S. Lilley, B. R. Fulton, M. A. Nagarajan, I. J. Thompson, and D. W. Banes, Phys. Lett. 151B, 181 (1985).

[24] F. Videbaek, R. B. Goldstein, L. Grodzins, S. G. Steadman, T. A. Belote, and J. D. Garrett, Phys. Rev. C 15, 954 (1977); E. Vulgaris, L. Grodzins, S. G. Steadman, and R. Ledoux, ibid. 33, 2017 (1986); 341495 (1986); B. B. Back, R. R. Betts, J. E. Gindler, B. D. Winkins, S. Saini, M. B. Tsang, C. K. Gelbke, W. G. Lynch, M. A. McMahan, and P. A. Baisden, ibid. 32, 195 (1985); T. Murakami, C.-C. Sahm, R. Vandenbosch, D. D. Leach, A. Ray, and M. J. Murphy, ibid. 34, 1353 (1986).

[25] J. A. Ruiz, J. L. Ferrero, B. Bilwes, and R. Bilwes, Nucl. Phys. A548, 510 (1992).

[26] D. L. Hendrie, Phys. Rev. C 31, 478 (1973).

[27] M. Beckerman, M. Salomaa, A. Sperduto, and J. D. Molitoris, Phys. Rev. C 25, 837 (1982).

[28] G. R. Satchler, Direct Nuclear Reactions (Oxford University Press, New York, 1983).

[29] D. Schwalm, E. K. Warburton, and J. W. Olnew, Nucl. Phys. A293, 425 (1977).

[30] R. H. Spear, Phys. Rep. 73, 369 (1981). 